\title{
Should a driving support agent provide explicit instructions to the user? Video-based study focused on politeness strategies
}

\author{
Tomoki Miyamoto \\ Graduate school of Tokyo Polytechnic \\ University, JSPS Research Fellowship \\ for Young Scientists \\ d1985001@st.t-kougei.ac.jp \\ Hitoshi Kanamori \\ Institute of Innovation for Future \\ Society of Nagoya University \\ hitoshi_kanamori@coi.nagoya- \\ u.ac.jp
}

\author{
Daisuke Katagami \\ Tokyo Polytechnic University \\ katagami@t-kougei.ac.jp \\ Yuki Yoshihara \\ Institute of Innovation for Future \\ Society of Nagoya University \\ y-yuki@coi.nagoya-u.ac.jp
}

\author{
Takahiro Tanaka \\ Institute of Innovation for Future \\ Society of Nagoya University \\ tanaka@coi.nagoya-u.ac.jp
}

Kazuhiro Fujikake

Chukyo University

fujikake@lets.chukyo-u.ac.jp

\begin{abstract}
Soon, autonomous cars are expected to become widespread, but at present, it is still common for people to drive cars manually. A driving support agent (DSA) is used to support driving using the human-agent interaction approach. In previous research, it was shown that DSAs can be useful in assisting the user through voice utterances. However, previous studies focusing on DSA utterance design have not compared off-record strategies with other politeness strategies that explicitly communicate the speaker's intentions, and it was not clear whether DSA should provide explicit utterances to the user. Therefore, in this study, a video-based subjective evaluation experiment $(n=240)$ was conducted to compare the acceptability of off-record strategies, positive politeness strategies, negative politeness strategies, and direct utterances without politeness strategies. The results of the experiment showed that the negative politeness strategy was evaluated significantly higher than the off-record strategy on evaluation items related to functionality. This result suggests the usefulness of providing explicit instructions with linguistic consideration (politeness) when DSA provides driving assistance to users. In addition to the above findings, there were several correlations between the user's personality characteristics and the subjective evaluation of the DSA. Specifically, there was a significant positive correlation between the level of conscientiousness of the user and the direct utterance evaluation value. For the positive politeness strategy and off-record strategy, there were no significant correlations between evaluation categories of the dislikeability and users' personality characteristics. These results suggest that individual differences in negative impressions due to personality characteristics are small in the positive politeness and off-record strategies.
\end{abstract}

Permission to make digital or hard copies of all or part of this work for personal or classroom use is granted without fee provided that copies are not made or distributed for profit or commercial advantage and that copies bear this notice and the full citation on the first page. Copyrights for components of this work owned by others than ACM must be honored. Abstracting with credit is permitted. To copy otherwise, or republish, to post on servers or to redistribute to lists, requires prior specific permission and/or a fee. Request permissions from permissions@acm.org.

HAI '21, November 09-11, 2021, Virtual Event, Japan

(c) 2021 Association for Computing Machinery.

ACM ISBN 978-1-4503-8620-3/21/11 \$15.00

https://doi.org/10.1145/3472307.3484160

\section{CCS CONCEPTS}

- Human-centered computing; • Human computer interaction (HCI); • HCI design and evaluation methods; • User studies.;

\section{KEYWORDS}

driving support agent, politeness strategies, acceptability, personality

\section{ACM Reference Format:}

Tomoki Miyamoto, Daisuke Katagami, Takahiro Tanaka, Hitoshi Kanamori, Yuki Yoshihara, and Kazuhiro Fujikake. 2021. Should a driving support agent provide explicit instructions to the user? Video-based study focused on politeness strategies. In Proceedings of the 9th International Conference on Human-Agent Interaction (HAI '21), November 09-11, 2021, Virtual Event, Japan. ACM, New York, NY, USA, 11 pages. https://doi.org/10.1145/3472307. 3484160

\section{INTRODUCTION}

In recent years, widespread use of autonomous cars has been expected, but it is still common to drive a car manually. Because accidents can occur due to human error in manual driving, there is a strong need to support driving with engineering technology. A driving support agent (DSA) is used as an engineering support method for manual driving (Figure 1). Previous studies on DSA [13] have shown the effectiveness of small dashboard robots, which assist the driver through voice utterances. In addition, research on DSA is a hot topic in the human-agent interaction (HAI) community, and a study of DSA [3] won the Best Paper Award at the International Conference on HAI 2017.

The main utterances of previous DSAs [1-3] were designed to give implicit instructions to avoid causing discomfort to the user (e.g., the DSA says "there is a pedestrian" and does not specify the driving behaviors the user should perform). According to politeness theory [4], a knowledge in the research field of sociolinguistics and pragmatics, such implicit instruction corresponds to the "hinting" strategy (off-record), which avoids directly communicating intentions to the other party. Nevertheless, previous studies $[5,6]$ that evaluated the acceptability of the politeness theory utterance strategies used by DSAs have focused only on strategies that convey explicit intentions, and the difference between the effects of these 


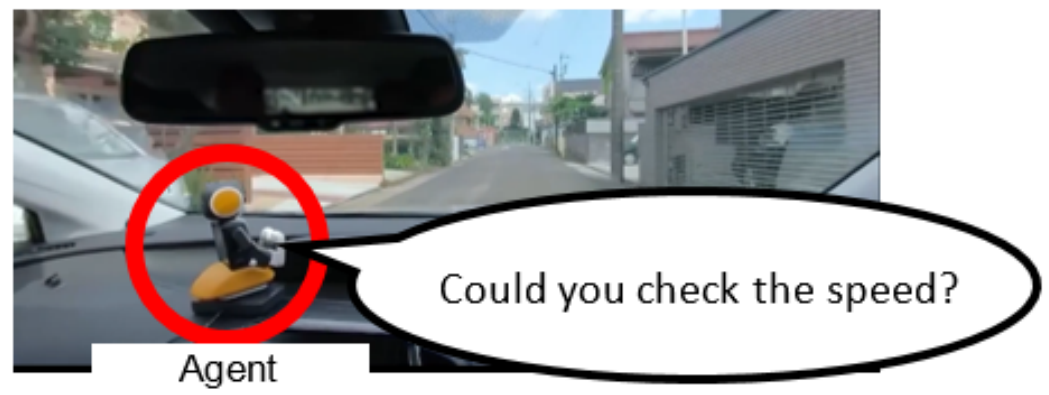

Figure 1: A DSA and example of utterance

and off-record strategies was not clear. In other words, it is unclear whether the DSA should explicitly instruct the user on driving operations. Thus, in this study, we set a research question (RQ), "Should a driving support agent provide explicit instructions to the user?". Based on previous studies [1, 2], the theoretical hypothesis of this study is "ORS is more acceptable than explicit strategies because it does not provide explicit instructions to users."

The purpose of this study is to evaluate the acceptability of explicit and implicit instructions uttered by the DSA on the driving behavior that the user should perform and to validate the RQ. For this purpose, the acceptability of utterances without politeness strategy (i.e., direct, DRC), with positive politeness strategy (PPS), with negative politeness strategy (NPS), and with off-record strategy (ORS) will be compared in a video-based subjective evaluation experiment using the strategy of utterance (wording) used by DSA as an independent variable. In addition, since the acceptability of DSA utterances is thought to vary depending on the personality characteristics of the user, we analyzed the relationship between the acceptability of the utterance strategy and the personality characteristics of the user. As a dependent variable, we will use DSA acceptability evaluation measures $[1,2]$ : the better the results of the evaluation measures $[1,2]$, the higher the acceptability of the DSA by the users. The working hypothesis is that "the subjective evaluation of ORS will be higher than the evaluation of other strategies." In addition, owing to individual differences in the acceptability of driver assistance, we expect that the acceptability will be correlated with the personality characteristics of the user. Previous research on DSA includes experiments with driving simulators and actual cars, but with such empirical approaches, ensuring a large sample size (e.g., $\mathrm{n}>200$ ) incurs significant costs. However, to discuss the acceptability of DSA at a finer level, such as the relationship between the acceptability of DSA and the user's personality characteristics, it is necessary to conduct not only an empirical experiment but also an investigation with a large sample size, as in this study. In addition, in this study, the participants of the experiment were native speakers of Japanese, because the need for DSAs is considered to be particularly high in Japan [1-3].

The remainder of this paper is organized as follows: In Section 2, we describe related work on politeness strategy, politeness in DSAs and other agents, and the relationship between the user's personality characteristics and acceptability of agents. In Section 3 , we describe the video-based evaluation of the DSA to verify the RQ.
In Sections 4 and 5, we present the results and their significance. Finally, the conclusions of this paper are presented in Section 6.

\section{RELATED WORK}

\subsection{Politeness strategies}

Politeness strategies are utterance strategies (linguistic considerations) that facilitate good human relations, as defined in the politeness theory by Brown and Levinson [4]. Politeness theory assumes a conversation between two parties, and the speaker (S) estimates the face (desire in human relations) of the hearer $(\mathrm{H})$ as a criterion for choosing politeness strategies.

- Positive Face (PF): H's desire for psychological closeness with S.

- Negative Face (NF): H's desire to maintain a psychological distance from $\mathrm{S}$ (neither approaching nor leaving).

$\mathrm{S}$ estimates whether $\mathrm{H}$ has a PF or NF; S chooses PPS if they estimate that $\mathrm{H}$ has a PF, and NPS if they estimate that $\mathrm{H}$ has an NF. There is also a case in which S expects that using either PPS or NPS will result in a large threat to H's face. In this case, $\mathrm{S}$ chooses ORS. Because ORS does not convey explicit intentions to $\mathrm{H}$, it is effective in reducing the risk of threatening H's face [4]. Equation 1) has been defined as a criterion for $S$ to estimate the face of $H$ [4].

$$
W x=D(S, H)+P(H, S)+R x
$$

In Eq. 1), $\mathrm{D}(\mathrm{S}, \mathrm{H})$ represents the social distance between $\mathrm{S}$ and $\mathrm{D}, \mathrm{P}(\mathrm{H}, \mathrm{S})$ represents the amount of social power (difference in position) of $\mathrm{S}$ over $\mathrm{H}$, and $\mathrm{Rx}$ represents the degree of threat of action $\mathrm{x}$ itself in a particular situation or culture. $\mathrm{S}$ estimates the degree to which action $\mathrm{x}$ threatens the face of $\mathrm{H}(\mathrm{Wx})$ by summing up these three variables. In this paper, DSA is $\mathrm{S}$ and user is $\mathrm{H}$; thus, Eq. 2) is valid.

$$
W x=D(D S A, U \text { ser })+P(U \operatorname{ser}, D S A)+R x
$$

In Eq. 2), $\mathrm{x}$ is the driving support by DSA.

\subsection{Politeness research on agents}

Miyamoto et al. [5, 6] and Lee et al. [7] partially verified the effects of politeness strategies in DSA. In the study by Miyamoto et al. [5, 6], the effects of PPS and NPS were compared, and PPS was evaluated significantly higher than NPS. In contrast, it was shown that there are some users who prefer NPS to PPS [5]. The study of Lee et 
al. [7] showed the effectiveness of DSA that used NPS. However, previous DSA studies $[1,2]$ primarily adopted utterances that fell under ORS, although they did not refer to politeness theory [4]. This design increases acceptability by not giving explicit instructions to the user [2]. In addition, in the study of Miyamoto et al. [5, 6] of DSA utterance design for Japanese, politeness strategy and sentence-final style (Japanese honorific system) are treated as one variable. This is because the literature [8] suggests that sentencefinal styles in Japanese have similar effects to politeness strategies [5]. Specifically, formal sentence-final styles (e.g., in conversations with superiors) and NPS as well as informal sentence-final styles (e.g., in conversations among friends) and PPS have similar effects [8]. However, PPS utterances with a formal sentence-final style and NPS utterances with an informal sentence-final style are also commonly used in Japanese conversation [8]. Thus, to discuss the utterance design of DSA more accurately in Japanese, it is useful to clarify the acceptability of DSA when the sentence-final style is manipulated as a variable independent of the politeness strategy. Therefore, in this study, politeness strategies and sentence-final style were manipulated as separate variables. The details of the experimental factors are described in Section 3.1.

Politeness strategies have also been applied in the study of agents other than DSA. For example, PPS is more effective than NPS in situations where a humanoid robot asks for help from the user [9], and the study of Torrey et al. [10] applied politeness theory to the utterance of a robot giving cooking advice, suggesting the effectiveness of NPS. In a study assuming a chat between a virtual agent and a person, it was reported that while the agent's use of PPS can reduce the impression of machine-ness (have no intention), the overall impression is higher with NPS [11]. In a study of multiparty conversations, in a task in which virtual agents conducting a multiparty conversation invite a user to join the conversation, the probability of the user joining the conversation was significantly increased when the agents used PPS [12]. Although these findings are useful for designing smooth interactions between people and agents, they are not necessarily applicable to other situations, as both studies were conducted for specific situations. In the same way that the appropriate behavior for human interaction differs depending on the situation, it is necessary to design and evaluate the agent's utterance according to the agent's role. Therefore, it is necessary to conduct experiments that focus on DSA.

\subsection{User personality and acceptability of agents}

With regard to the influence of user personality characteristics on the acceptability of DSA, studies aimed at reducing the anger of users who drive cars [13] have shown that personality characteristics are useful for predicting user preferences for the methods by which the system intervenes with them (visual, tactile, auditory). Previously, Jeon et al. [14] showed that voice-based DSA was effective in reducing user anger, but no analysis focused on individual differences in the personality characteristics of the user. However, the previous study [13] used a large-scale questionnaire survey that also covered methods other than DSA (e.g., music, radio, funny videos, etc.), and DSA utterances were not used in the experiment as stimuli. In this study, we used videos in which DSA speaks utterances as experimental stimuli. In addition, although there have been studies to add human-like personalities to DSAs [15], the purpose and approach are different from this study, which focuses on the personality characteristics of users.

In addition to the above, user personality characteristics have been applied in the design of agents that interact with humans. For example, a method has been developed to estimate the appropriate behavior of a conversational system for customer relationship management based on the customer's personality characteristics [16], and the influence of the user's personality characteristics on the acceptability of compliments by a humanoid robot has been analyzed [17]. In a study [18] that investigated the tendency of users to anthropomorphize robots, it was shown that users with high extraversion were more likely to perceive robots as anthropomorphic. It has also been suggested that the user's personality characteristics affect the level of trust in the machine in cooperative tasks with high cognitive load and uncertainty [19], and that it is important to consider the user's personality characteristics in the design of robots that provide health care to the user [20]. Although these findings may be partially applicable to DSA, we investigate DSA-specific knowledge because assisting car driving is an important task in relation to users' lives.

\section{MATERIALS AND METHODS}

In this section, we describe a video-based evaluation experiment of DSA utterance strategies to verify the RQ "Should a driving support agent provide explicit instructions to the user?" This experiment was conducted with the approval of the research ethics committee of the organization to which the authors belong.

\subsection{Experimental design and stimuli}

In this experiment, the utterance strategy was set as betweensubject factor. The experimental levels were set by referring to the politeness theory [4] and experimental designs of previous studies that applied politeness theory to agents $[5,6,9]$ :

- Direct (DRC) level: The DSA does not use a politeness strategy but provides explicit instructions on car driving behavior. If the evaluation of this level is the highest in the experiment, it would suggest that the DSA does not need to use politeness strategies.

- Positive Politeness Strategy (PPS) level: The DSA provides explicit instructions while using phrases that include both the user and the DSA in the car driving behavior.

- Negative Politeness Strategy (NPS) level: The DSA explicitly states the behavior the user should adopt but phrases it indirectly in such a way that the user has room to refuse it.

- Off-record strategy (ORS) level: DSA does not explicitly state what behavior the user should adopt but uses implicit utterances to give hints. The DSAs in previous studies [1-3] applied this level.

Furthermore, the sentence-final style factor (formal/informal) was set as a within-subject factor in this experiment. That is, for each utterance strategy level, two patterns of utterances were set (a mixed design of $4 \times 2$ ): formal (as if talking to a superior) and informal (as if talking to a friend).

Based on the above experimental conditions, we created experimental stimuli (videos). To create the experimental stimuli, we used an 


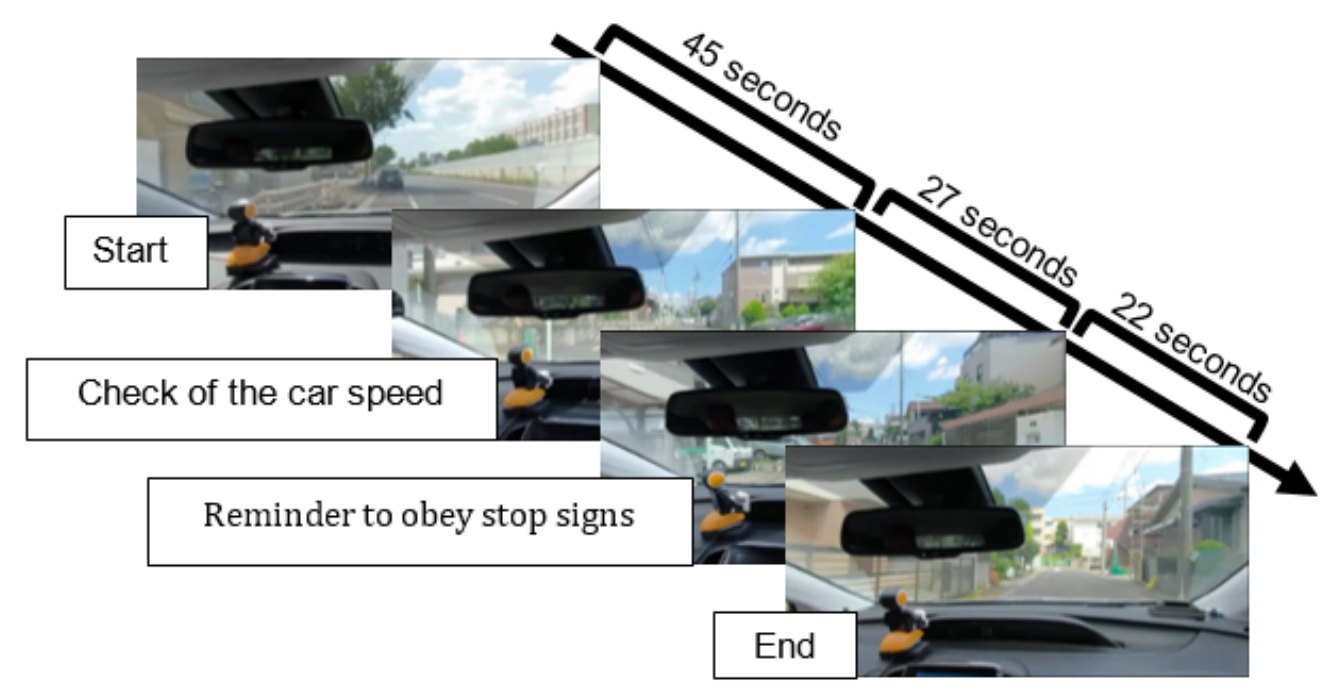

Figure 2: Flow of the experimental stimuli. First, the car goes straight for a while, makes two left turns, and enters a residential area, where the DSA gives the first utterance (instruction to check the speed). After that, the car goes straight for a while again and the DSA speaks for the second time (to alert the driver to pause). After the car pauses, it goes straight for a while, and the movie ends.

Table 1: Utterances of DSA in the experimental videos (fDRC, iDRC, fPPS, and iPPS). The bold shows a formal style.

\begin{tabular}{|c|c|c|c|c|}
\hline & fDRC & iDRC & fPPS & iPPS \\
\hline Check of the car speed & $\begin{array}{l}\text { Koko ha nan kiro seigen } \\
\text { desyo u ka? Sokudo wo } \\
\text { kakunin si te kudasai. }\end{array}$ & $\begin{array}{l}\text { Koko ha nan kiro seigen } \\
\text { ka naa? Sokudo wo } \\
\text { kakunin si te. }\end{array}$ & $\begin{array}{l}\text { Koko ha nan kiro seigen } \\
\text { desyo u ka? Sokudo wo } \\
\text { kakunin si masyo u. }\end{array}$ & $\begin{array}{l}\text { Koko ha nan kiro seigen } \\
\text { ka naa? Sokudo wo } \\
\text { kakunin siyo u. }\end{array}$ \\
\hline English & \multicolumn{2}{|c|}{ What is the speed limit here? Check the speed. } & \multicolumn{2}{|c|}{ What is the speed limit here? Let's check the speed. } \\
\hline $\begin{array}{l}\text { Reminder to obey stop } \\
\text { signs }\end{array}$ & $\begin{array}{l}\text { Mousugu itizi teisi desu } \\
\text { ne. Gensoku si te teisi si } \\
\text { te kudasai. }\end{array}$ & $\begin{array}{l}\text { Mousugu itizi teisi da ne. } \\
\text { Gensoku si te teisi si te. }\end{array}$ & $\begin{array}{l}\text { Mousugu itizi teisi desu } \\
\text { ne. Gensoku si te teisi si } \\
\text { masyo u. }\end{array}$ & $\begin{array}{l}\text { Mousugu itizi teisi da ne. } \\
\text { Gensoku si te teisi siyo u. }\end{array}$ \\
\hline English & \multicolumn{2}{|c|}{$\begin{array}{l}\text { There is a stop sign immediately ahead. Slow down and } \\
\text { stop. }\end{array}$} & \multicolumn{2}{|c|}{$\begin{array}{l}\text { There is a stop sign immediately ahead. Let's slow } \\
\text { down and stop. }\end{array}$} \\
\hline
\end{tabular}

existing video (original video) in which the DSA provides support to the user in a car. The original video was provided by the Nagoya University driver support research project through an agent and was filmed during a test run of DSA on public roads; the DSA was developed based on the results of previous research [2,3]. Tables 1 and 2 show the DSA utterances for each experimental level based on the driving situations in the original video. The DRC, PPS, and NPS utterances are structured so that an ORS utterance is followed by an utterance using each strategy. In other words, in experimental levels other than ORS, the DRC, PPS, and NPS utterances are added to the ORS utterances (implicit instructions), so that the utterance becomes an explicit instruction. This is because, for example, if the DSA only utters "Could you slow down and stop?" as formal NPS (fNPS), the user may not understand why they should slow down and stop and may become confused. In addition, we focus on the DSA instructions regarding the driving behavior that the user should perform. Thus, we adopted the aforementioned structure of utterances as the difference between the ORS and the other experimental levels in terms of whether the DSA explicitly identifies the behavior that the user should perform. In the original video, the DSA speaks about checking the car speed and reminds the driver to obey stop signs. In this experiment, the DSA spoke in the same situations and with the same timing as in the original video. The experimental stimuli were created by editing the DSA utterances in the original video. Figure 2 shows the flow of the experimental stimuli (video). The voice of the DSA in the experimental videos was the same as that of the agent $\left(\mathrm{RoBoHoN}^{1}\right)$ used in the original video. The DSA voice is simulated as a male child1. The results of previous studies [1] show that the appearance and voice of RoBoHoN are appropriate for DSA.

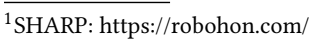


Table 2: Utterances of DSA in the experimental videos (fNPS, iNPS, fORS, and iORS).

\begin{tabular}{|c|c|c|c|c|}
\hline & fNPS & iNPS & fORS & iORS \\
\hline $\begin{array}{l}\text { Check of the } \\
\text { car speed. }\end{array}$ & $\begin{array}{l}\text { Koko ha nan kiro seigen } \\
\text { desyo u ka? Sokudo wo } \\
\text { kakunin deki masu ka? }\end{array}$ & $\begin{array}{l}\text { Koko ha nan kiro seigen ka } \\
\text { naa? Sokudo wo kakunin } \\
\text { dekiru? }\end{array}$ & $\begin{array}{l}\text { Koko ha nan kiro seigen } \\
\text { desyo u ka? }\end{array}$ & $\begin{array}{l}\text { Koko ha nan kiro seigen ka } \\
\text { naa? }\end{array}$ \\
\hline English & What is the speed limit her & ? Could you check the speed? & What is the speed limit here? & \\
\hline $\begin{array}{l}\text { Reminder to } \\
\text { obey stop } \\
\text { signs. }\end{array}$ & $\begin{array}{l}\text { Mousugu itizi teisi desu } \\
\text { ne. Gensoku si te teisi } \\
\text { deki masu ka? }\end{array}$ & $\begin{array}{l}\text { Mousugu itizi teisi da ne. } \\
\text { Gensoku si te teisi dekiru? }\end{array}$ & Mousugu itizi teisi desu ne. & Mousugu itizi teisi da ne. \\
\hline English & \multicolumn{2}{|c|}{$\begin{array}{l}\text { There is a stop sign immediately ahead. Could you slow } \\
\text { down and stop? }\end{array}$} & \multicolumn{2}{|c|}{ There is a stop sign immediately ahead. } \\
\hline
\end{tabular}

\subsection{Evaluation items of DSA acceptability}

In this experiment, we adopted the psychological scale used in studies that evaluated the acceptability of DSA [1,2]. For ease of analysis, the nine items in this scale $[1,2]$ were classified into the following three categories.

Category 1: Likeability includes favorability, reliability, and familiarity.

Category 2: Dislikeability includes the items of discomfort, annoyance, and disturbance. The items in this category are reverse items.

Category 3: Functionality includes the desire to use, usability, and contribution to safe driving.

The participants responded to each item on a seven-point Likert scale (1: Strongly disagree, 4: Neither, and 7: Strongly agree) after viewing the video of the experimental stimuli. The evaluation score for each category is the total score of the three items in the category (max is 21 points). Thus, the closer the evaluation value was to 21 points, the more acceptable the DSA is in the evaluation categories (i.e., The DSA kept the user's face). Among the three categories, likeability and dislikeability are similar, but likeability measures the strength of the positive (good) impression of the DSA, whereas dislikeability measures the strength of the negative (bad) impression of the DSA. The internal consistency of each evaluation category is described in Section 4.1.

\subsection{Measurement of personality characteristics}

In this experiment, the Ten Item Personality Inventory (TIPI) [21] was adopted to measure the personality characteristics of the participants. The TIPI can measure the five personality characteristics (extraversion, agreeableness, conscientiousness, emotional stability, and openness to experience) with 10 questions. Since the participants in this experiment were native speakers of Japanese, the Japanese version of the TIPI [22] was used. Participants evaluated themselves on a total of 10 items (1: Extraverted, enthusiastic; 2: Critical, quarrelsome; 3: Dependable, self-disciplined; 4: Anxious, easily upset; 5: Open to new experiences, complex; 6: Reserved, quiet; 7: Sympathetic, warm; 8: Disorganized, careless; 9: Calm, emotionally stable; 10 : Conventional, uncreative) on a seven-point Likert scale (1. Strongly disagree, 4 . Neither agree nor disagree, and 7. Strongly agree). The adopted scoring method for each factor is based on that described in [21,22].

\subsection{Participants}

Participants were recruited using the crowdsourcing service CrowdWorks $^{2}$, and 240 evaluations (114 men and 126 women, mean age 40.1 years, $S D=9.6$ ) were analyzed. We verified with a questionnaire that all participants were native speakers of Japanese and had a driver's license. The breakdown of the participants in each experimental condition is presented in Table 3

To exclude unreliable evaluation data from the analysis, first, we required that the participant work approval rate on CrowdWorks was $95 \%$ or higher as a condition for participation. The work approval rate is an evaluation index of worker reliability defined in CrowdWorks and is calculated as "the number of tasks approved / the number of tasks done". In addition, the number of times a worker could participate in the experiment was set to one. Under these conditions, we manually extracted the data with obvious problems: we did not approve the work if the questionnaire was answered more than twice because it did not comply with the recruitment conditions. Based on this, we excluded the data of eight participants from the analysis. Then, we re-advertised to substitute the evaluation data of the eight cases. As a result, eight new evaluations were collected and included in the analysis. Workers were informed in writing about the possibility that their work would not be approved if they did not comply with the conditions of the recruitment process before they were allowed to participate in the experiment, and then they agreed to participate in the experiment. Participants whose work was approved received an honorarium at the end of the experiment.

\subsection{Procedure}

Figure 3 shows an outline of the experimental procedure. First, participants viewed a text-based briefing about the experiment on a questionnaire platform (Google forms ${ }^{3}$ ) and freely decided whether to participate. Then, the participants entered their worker ID, gender, age, and answered the TIPI-J questionnaire. After that, participants were given text-based instructions on how to watch the experimental videos. The important points of the instructions for the participants were as follows:

1. Participants should watch the video as if they were the driver.

\footnotetext{
${ }^{2}$ Crowd Works: https://crowdworks.jp/

${ }^{3}$ Google forms: https://www.google.com/intl/ja_jp/forms/about/
} 
Table 3: Breakdown of participants in the experiment.

\begin{tabular}{llllll}
\hline & All of number & Male & Female & Means of age & SD of age \\
\hline DRC & 59 & 37 & 22 & 39.8 & 8.0 \\
PPS & 61 & 17 & 44 & 39.6 & 10.5 \\
NPS & 60 & 33 & 27 & 39.9 & 9.0 \\
ORC & 60 & 27 & 33 & 41.1 & 10.0 \\
\hline
\end{tabular}

\begin{tabular}{|c|c|c|}
\hline $\begin{array}{c}\text { Read the explanation of } \\
\text { the experiment }\end{array}$ & Response to TIPI-J & $\begin{array}{l}\text { Read the instructions for } \\
\text { watching the videos }\end{array}$ \\
\hline $\begin{array}{c}\text { Watch Video A } \\
\text { (e.g, fNPS) }\end{array}$ & Evaluate Video A & $\begin{array}{c}\text { Watch Video B } \\
\text { (e.g, iNPS) }\end{array}$ \\
\hline Evaluate Video B & Submit Results & Finish \\
\hline
\end{tabular}

Figure 3: Flow of the experimental procedure.

2. The DSA speaks twice to the participants in the video. After the car makes two left turns, the DSA speaks for the first time. After that, it goes straight for a while, and then the DSA speaks the second time.

3. Participants were assumed to use the DSA (RoBoHoN) for the first time when watching the video. Even if they have used RoBoHoN on other occasions, they should think of that RoBoHoN and the RoBoHoN in the video as different entities and watch the video as if they were using it for the first time.

4. Participants watched and evaluated the videos as if the RoBoHoNs in Videos A and B were completely different entities.

The purpose of instruction 2 was to prevent the participants from feeling anxious about the fact that the DSA does not speak for a while. For instruction 3, we reminded with text the participants just before they watched the video so that there would be no bias in their evaluation. After confirming the above instructions, participants watched the experimental videos. After watching Video A (formal or informal level), participants evaluated the acceptability of DSA in Video A based on the scale described in Section 3.2. Then, they watched and evaluated the DSA in Video B (different sentence-final style from Video A). To cancel the order effect of the sentence-final style factor, the order in which the videos were viewed differed among participants. In this experiment, 121 participants watched the formal style level video as Video A, and 119 participants watched the informal style level video as Video A.

\section{RESULTS}

\subsection{Internal consistency checks of acceptability metrics}

The validity of the acceptability categories in Section 3.2, was investigated using Cronbach's alpha coefficient. The alpha coefficients were calculated for the within-subjects factor, and they were high at all experimental levels, with the lowest value being 0.86 . Thus, the internal consistency of each evaluation category is sufficient.

\subsection{Evaluation results of acceptability based on the three categories}

A two-way ANOVA was employed to analyze the evaluation results, with a significance level of $5 \%$. If a significant difference (significant tendency) was found at the $10 \%$ level, although the null hypothesis was not rejected, it was used as a reference for discussion. Figure 4 (left side) shows the evaluation results of likability. For all the utterance strategies, the evaluation value of the formal sentence-final style was higher than that of the informal sentence-final style. A two-way ANOVA showed a significant main effect for the sentencefinal style factor $\left(\mathrm{F}(1,236)=21.79, \mathrm{p}<0.01, \eta^{2}=0.018\right)$. The notation for the significant difference in the legend of the graph indicates that the main effect of the sentence-final style factor was significant. However, the main effect of the utterance strategy factor $\left(\mathrm{F}(3,236)=1.64, \mathrm{p}>0.1, \eta^{2}=0.017\right)$ and the interaction between the experimental factors were not significant $(\mathrm{F}(3,236)=1.52, \mathrm{p}>$ $\left.0.1, \eta^{2}=0.003\right)$. Similarly, in the evaluation results of dislikeability, there was a main effect of the sentence-final style factor $(\mathrm{F}(1,236)=$ $\left.30.56, \mathrm{p}<0.01, \eta^{2}=0.021\right)$, whereas the main effect of the utterance strategy factor $\left(\mathrm{F}(3,236)=0.18, \mathrm{p}>0.1, \eta^{2}=0.001\right)$ and the interaction between factors were not significant $(\mathrm{F}(3,236)=0.25, \mathrm{p}>0.1$, $\left.\eta^{2}=0.002\right)$. The interaction between the utterance strategy factor and the sentence-final style factor tended to be significant $(\mathrm{F}(3$, 236) $\left.=2.12, \mathrm{p}<0.1, \eta^{2}=0.003\right)$ in the evaluation of functionality scores (right side of Figure 4). In addition, the simple main effect of the utterance strategy factor at the formal level tended to be significant $\left(\mathrm{F}(3,307)=2.58, \mathrm{p}<0.1, \eta^{2}=0.016\right)$. Thus, there was a possibility that the evaluation values among the utterance strategy factors were significantly different at the formal level, and multiple comparisons using the Tukey method were conducted as a post hoc test. The results showed that the fNPS had a significant tendency $(p<0.1)$ to have higher scores for functionality than the PPPS, and the fNPS had significantly higher scores than the fORS $(p<0.01)$. In terms of utterance strategy factors, the simple main effect of the sentence-final style factor was significant at the NPS $(F(1,236)=$ 

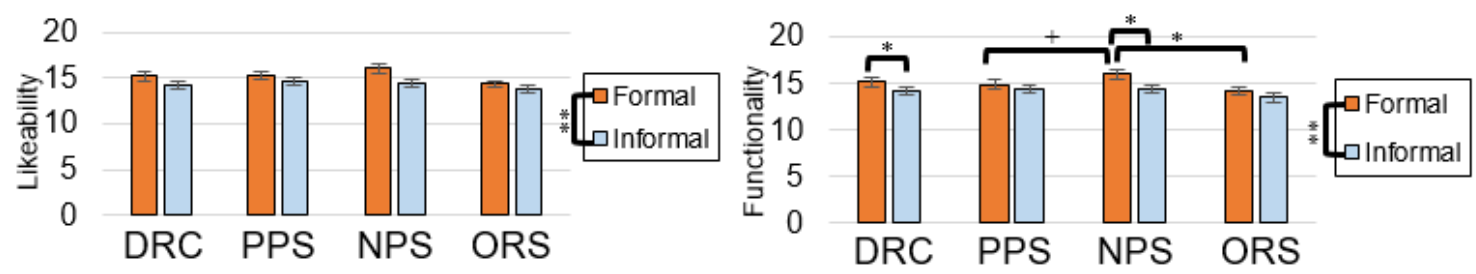

Figure 4: Evaluation results of the likeability (left) and functionality (right). The error bar shows the standard error. ${ }^{* *}: \mathrm{p}<$ $0.01,{ }^{*}: p<0.05,+: p<0.1$. The results for dislikeability were similar to those for likeability, so the graphs were omitted.

Table 4: Partial correlation coefficients between likeability and personality characteristics. ${ }^{* *}: \mathbf{p}<0.01,{ }^{*}: \mathbf{p}<0.05$.

\begin{tabular}{llllll}
\hline & Extraversion & Agreeableness & Conscientiousness & Emotional Stability & $\begin{array}{l}\text { Openness to } \\
\text { Experience }\end{array}$ \\
\hline f(over all) & 0.06 & $\mathbf{0 . 1 5}^{*}$ & 0.10 & 0.04 & 0.00 \\
fDRC & -0.03 & 0.16 & $\mathbf{0 . 4 3}^{* *}$ & 0.06 & -0.15 \\
iNPS & -0.11 & $\mathbf{0 . 2 7}^{*}$ & 0.02 & 0.13 & $\mathbf{- 0 . 3 9}^{* *}$ \\
fORS & -0.10 & $\mathbf{0 . 3 6}^{* *}$ & -0.08 & 0.07 & $\mathbf{0 . 2 7}^{*}$ \\
\hline
\end{tabular}

Table 5: Partial correlation coefficients between dislikeability and personality characteristics. ${ }^{*}: \mathbf{p}<0.01,{ }^{*}: \mathbf{p}<0.05$.

\begin{tabular}{llllll}
\hline & Extraversion & Agreeableness & Conscientiousness & Emotional Stability & $\begin{array}{c}\text { Openness to } \\
\text { Experience }\end{array}$ \\
\hline f(over all) & 0.05 & 0.02 & $0.18^{* *}$ & 0.00 & -0.01 \\
fDRC & 0.00 & 0.21 & $0.47^{* *}$ & 0.06 & -0.06 \\
iNPS & 0.01 & 0.13 & 0.04 & 0.05 & $-0.39^{* *}$ \\
\hline
\end{tabular}

22.32, $\left.\mathrm{p}<0.01, \eta^{2}=0.012\right)$ and $\mathrm{DRC}\left(\mathrm{F}(1,236)=7.15, \mathrm{p}<0.01, \eta^{2}=\right.$ $0.004)$, and the formal level was evaluated significantly higher than the informal level at the NPS and DRC $(\mathrm{p}<0.01)$.

\subsection{Correlation between DSA acceptability and user personality characteristics}

Partial correlation coefficient analysis was adopted as a method to analyze the relationship between the acceptability of DSA and the user's personality characteristics. By using partial correlation analysis, we can obtain results that remove the influence of the third factor that causes pseudo-correlation. Therefore, the partial correlation coefficient ( $\mathrm{r}$ ) tends to have a smaller effect size than the usual methods of analyzing correlation when the influence of the third factor is large, but the reliability of the results obtained is high. We calculated the partial correlation coefficients between the user's personality characteristics and the acceptability of DSA in each of the three evaluation categories. To test whether the calculated partial correlation coefficients were significant, we conducted a test of uncorrelation at the same significance level as in Section 4.2. The partial correlation coefficients for each evaluation category and personality characteristics are shown in Tables 4-6. The experimental levels not listed in each table are those that showed no significant correlation with the user's personality characteristics. The results show that there are significant correlations between the likeability evaluation of DSA and user agreeableness, conscientiousness, and openness to experience, between the dislikeability evaluation and users' extraversion, agreeableness, and conscientiousness, and between the functionality evaluation of DSA and users' conscientiousness and openness to experience. In addition, the sizes of $r$ were small to moderate. A detailed discussion of these results is provided in Section 5.2.

\section{DISCUSSION}

\subsection{Should a driving support agent provide explicit instructions to the user?}

The results suggest that it is useful to provide explicit instructions to users while taking linguistic considerations into account to increase the impression of the functionality of DSA. This result is contrary to our hypothesis, in terms of functionality. Specifically, at the formal sentence-final style level, the functionality scores were significantly higher for NPS than for ORS. The reason for these results may be that in the functionality category, the participants evaluated the DSA in terms its usefulness for driving. As fORS does not provide explicit instructions, we consider that the fORS utterances were not perceived as useful information for driving by the users; thus, their evaluation was lower than fNPS. We believe that the reason for the 
Table 6: Partial correlation coefficients between functionality and personality characteristics. ${ }^{* *}: \mathbf{p}<0.01,{ }^{*}: \mathbf{p}<0.05$.

\begin{tabular}{cccccc}
\hline & Extraversion & Agreeableness & Conscientiousness & Emotional Stability & $\begin{array}{c}\text { Openness to } \\
\text { Experience }\end{array}$ \\
\hline f(over all) & 0.08 & 0.10 & $\mathbf{0 . 1 3}^{*}$ & 0.07 & -0.02 \\
i(over all) & -0.04 & 0.09 & $\mathbf{0 . 1 3}^{*}$ & -0.02 & -0.05 \\
fDRC & 0.11 & -0.01 & $\mathbf{0 . 4 1}^{* *}$ & 0.00 & -0.21 \\
fNPS & $\mathbf{0 . 3 0}^{*}$ & 0.15 & -0.05 & 0.09 & 0.04 \\
fORS & -0.14 & $\mathbf{0 . 4 0}^{* *}$ & 0.06 & $0.23+$ & $0.25+$ \\
\hline
\end{tabular}

high evaluation of fNPS is that the expression "Could you ...?" in the NPS gives the user the option to refuse the instructions and increases acceptability. Although this result does not support our hypothesis, it contributes to the design of DSA utterances, since previous DSA studies [1-3] implemented utterances that fall under ORS. However, if a relationship is established between the DSA and the user or if the DSA provides repeated instructions to the user, different results may be observed. In addition, different results may be obtained depending on the appearance or voice of the DSA. However, since RoBoHoN was effective as the DSA in previous studies $[1,5,6]$, the results have some usefulness in the design of DSAs.

Other results showed a significant main effect of the sentencefinal style factor in all evaluation categories (likeability, dislikeability, and functionality), with formal sentence-final style being evaluated higher than informal style. In this experiment, participants were instructed to watch the video with the intention of using the DSA for the first time, so the relationship between the DSA and the user (i.e., D(DSA, User)) is the first meeting, which may have resulted in a higher evaluation of the formal style. These results support the results of the study of Lee et al. [7], which showed the effectiveness of DSA's polite utterances. In contrast, the results of Miyamoto et al. [5, 6], which compared the acceptability of DSA use of PPS and NPS, showed that PPS was evaluated significantly higher than NPS, which is different from the results of the present experiment. In these studies [5, 6], no instruction was given to the participants regarding their relationship with the DSA (D(DSA, User), Eq. 2)), and the interpretation of D(DSA, User) was left up to them. Therefore, the variance of D(DSA, User) assumed by the participants may be larger than in our experiment. We believe that by instructing the participants about D(DSA, User), and thus controlling the condition, we obtained more reliable results than previous studies $[5,6]$. Finally, the discussion in this section suggests that DSAs should use a formal sentence-final style in situations where they meet the user for the first time and should utter explicit instructions using NPS.

\subsection{Relationship between acceptability of DSA's politeness strategies and users' personality characteristics}

Several significant correlations were found between user personality characteristics and the acceptability of DSA in all three evaluation categories, which is consistent with our hypothesis. A common result across the three categories was a moderately significant positive correlation between the user's conscientiousness and fDRC evaluation value. A possible explanation of this result is that users with high conscientiousness may have interpreted the explicit and direct utterances of the DSA as accurate and sufficient information. Politeness theory [4] cites an utterance in life-threatening situations (e.g., disaster) as an example of how speakers utilize DRC. In the previous study [7], it was shown that the acceptability of polite utterances by the DSA was reduced when the car was breaking down (i.e., urgent). Since driving a car is always life-threatening, it is suggested that users with high conscientiousness (seriousness) may be more receptive to DRC regardless of the urgency of the driving situation.

There was a significant negative correlation between openness to experience and acceptability of iNPS, specifically likeability and dislikeability. It is said that people with high openness tend to think various thoughts based on the given information [23]. In this experiment, the NPS utterances used the question form "Could you...?" to be considerate of the user, creating more room for user interpretation compared to the DRC and PPS. Therefore, it is possible that the high openness to experience of the participants caused them to think various thoughts, and that they found the NPS annoying. In addition, there was no correlation between the user's personality characteristics and the dislikeability of PPS and ORS. This result suggests that the individual differences in negative impressions of DSA may be small for PPS and ORS. A previous study [24] suggested the importance of designing an agent that supports a user's task so that it is not rejected by the user, and the results of this experiment are useful for the design of DSA with small individual differences in acceptability. The above discussion suggests that it is important to consider the personality characteristics of users when designing DSA utterances. Since the fNPS, which was evaluated highly, did not show any negative correlation with personality characteristics, the fNPS is considered to have a high impression of functionality in the first meeting situation, regardless of the user's personality characteristics. However, NPS by DSA may not necessarily be highly acceptable to all users, because a weak negative correlation was found between users' openness to experience and the likeability/dislikeability of iNPS.

Finally, as a summary of this section, Table 7 shows the utterance strategies with the largest $r$ that showed a significant positive correlation $(p<0.05)$ with the user's personality characteristics in each evaluation category. From this table, it can be seen that there are utterance strategies that correspond to four personality characteristics other than emotional stability. These strategies have relatively high affinities with users who have high scores for each personality characteristic factor in this experiment. Although the 
Table 7: The utterance strategy has the highest positive correlation with the user's personality in each category. The results corresponding to $p<0.05$ were included. A "-" in the table indicates that there is no corresponding strategy.

\begin{tabular}{llllll}
\hline & Extraversion & Agreeableness & Conscientiousness & Emotional Stability & $\begin{array}{c}\text { Openness to } \\
\text { Experience }\end{array}$ \\
\hline Likeability & - & fORS & fDRC & - & fORS \\
Dislikeability & - & - & fDRC & - & - \\
Functionality & fNPS & fORS & fDRC & - & - \\
\hline
\end{tabular}

acceptability of ORS was not high in the evaluation by all participants (i.e., our hypothesis was not supported), it is suggested that users with high agreeableness and openness to experience tend to accept fORS.

\subsection{Potential contribution of the politeness study to HAI}

In this section, we discuss the usefulness of politeness in HAI research and its methodology. In the study of the HAI field, the humanity that users perceive in agents and the design of agents that users can easily accept are frequently discussed with reference to the theory of mind [25], intention stance [26], media equation [27], etc. In addition, the linguistic behavior of agents, which is the focus of this study, is one of the representative variables in HAI research [28]. Therefore, the application of a framework such as politeness theory [4], in which strategies to increase utility in human conversation are systematized, to HAI is considered to be a useful approach to discuss the humanity and acceptability of agents from the perspective of their verbal behavior. For example, in situations where an agent supports a user's life-critical task, it is useful for the agent to not only provide accurate information but also appropriately use linguistic considerations that are used in human conversation. However, a framework for discussing the relationships between different studies has not been established in the field of HAI research [28], and it is unclear to what extent the findings of this study apply to agents other than DSA. In addition, case studies of specific situations are often conducted in HAI research. Therefore, at present, politeness research in HAI requires individual utterance design and experimentation based on the role of the agent. However, we expect to be able to set up valid hypotheses in the future by using heuristics based on previous research and accumulated knowledge when designing new utterances for agents to which politeness research has not been applied. For example, the discussion on the user's personality characteristics and the agent's acceptability should be conducted in various other situations. The differences and the degree of agreement between the results obtained can be organized to facilitate the discussion on the conversational model of an agent that autonomously estimates the user's characteristics and speaks using politeness strategies according to them.

\subsection{Limitations and future work}

In this study, the experiment was conducted only with native Japanese speakers. However, there have been many discussions on cultural differences in HAI and human-robot interaction research [2931]. Thus, if a similar study is conducted with participants with different first languages different results may be obtained. We plan to investigate cultural and linguistic differences in the acceptability of politeness strategies used by agents in various situations, such as driving support and daily conversation. Investigating cultural differences is important as an approach to applying politeness theory to HAI because it examines the effect of varying Rx in Eq. 1) on agents' acceptability. In addition, this study did not deal with DSA, which provides information notifications to users in autonomous cars [32, 33]. DSA in autonomous cars needs to be discussed in separate research because the effective approach of assistance to users may differ depending on the autonomous driving level.

Because the experiments in this study were conducted under the assumption that the user and DSA meet for the first time, we did not conduct an evaluation of a situation in which the user and DSA have a previous relationship. In other words, $\mathrm{D}(\mathrm{S}, \mathrm{H})$ was controlled by the researchers in this study. In addition, $\mathrm{P}(\mathrm{H}, \mathrm{S})$ was not defined in a top-down manner. The relationship between the social power of the user and the DSA is considered to vary substantially depending on how the user perceives the DSA (e.g., as a tool or as a companion for cooperative work). For example, human driving instructors are considered by drivers to be as strict as teachers or superiors [34], but DSA may be simply a tool for the user. Therefore, it is possible that acceptable politeness strategies may vary depending on $\mathrm{P}(\mathrm{H}$, $\mathrm{S})$. In future research, it is necessary to discuss the effect of setting the relationship $\mathrm{D}(\mathrm{S}, \mathrm{H})$ differently than the first meeting on the acceptability of DSA, and the relationship between the tendency of user perception of the social position and power of artifacts and the acceptability of politeness strategies. The other limitation of this study is the granularity of the targeted politeness strategies. In politeness theory [4], 15 specific sub-strategies are defined for PPS, 10 for NPS, and 15 for ORS. Since the purpose of this paper was to evaluate explicit/implicit instructions in DSA utterances, we did not discuss the sub-strategies in detail, focusing on the amount of information possessed by PPS/NPS/ORS utterances and the degree of the threat to the user. In contrast, a study that selects strategies among the sub-strategies that are applicable to driving support and evaluates DSA utterances using all of these strategies is also possible. Such an approach will contribute to increasing the variety of useful utterances of the DSA.

The final goal of applying the politeness strategy to DSA is to realize a function that enables DSA to autonomously estimate the user's face in actual driving situations and to determine the utterance that is both desirable and helpful for safe driving. The effectiveness of our findings in actual driving situations needs to be verified through future experiments using driving simulators and actual cars. In addition, it is necessary to conduct evaluation 
experiments including multimodal information on factors related to mid-to-long-term interaction with the user, such as the frequency and timing of DSA speech, and on a more practical design of DSAs that can respond to the user's voice input, such as smart speakers [35]. In the research field of natural language processing, studies have been conducted to automatically estimate the politeness of sentences created by humans [36] and to automatically generate sentences with appropriate politeness [37], so it is possible that an utterance model can be constructed for DSA to autonomously provide appropriate support by referring to these studies [36, 37].

\section{CONCLUSION}

In this study, we set the RQ "Should a driving support agent provide explicit instructions to the user?" and evaluated the acceptability of politeness strategies in DSA utterances through a video-based study. In our hypothesis, we expected off-record strategy to be evaluated highly because it does not provide explicit instructions to the user. The results of the experiment showed that NPS with a formal sentence-final style was significantly higher in evaluation items related to the functionality of DSA. Although this result does not support our hypothesis, it shows the usefulness of DSA in providing explicit instructions while considering linguistic considerations (i.e., The answer to the research question is YES under the condition that the DSA uses NPS). For fNPS, which was evaluated highly for functionality, no negative correlation was found in any of the categories. Therefore, fNPS is considered to have a high impression of functionality, regardless of the user's personality characteristics.

\section{ACKNOWLEDGMENTS}

This work was partially supported by the Nagoya University Driver support research project through an agent.

\section{REFERENCES}

[1] Takahiro Tanaka, Kazuhiro Fujikake, Yuki Yoshihara, Nihan Karatas, Hirofumi Aoki, and Hitoshi Kanamori. 2020. Preliminary study for feasibility of driver agent in actual car environment -Driver agent for encouraging safe driving behavior (3). Journal of Transportation Technologies. 10, 2 (April 2020), 128-143. https://doi.org/10.4236/jtts.2020.102008

[2] Takahiro Tanaka, Kazuhiro Fujikake, Takashi Yonekawa, Makoto Inagami, Fumiya Kinoshita, Hirofumi Aoki, and Hitoshi Kanamori. 2018. Effect of difference in form of driving support agent to driver's acceptability -Driver agent for encouraging safe driving behavior (2). Journal of Transportation Technologies. 8, 3 (July 2018), 194-208. https://doi.org/10.4236/jtts.2018.83011

[3] Takahiro Tanaka, Kazuhiro Fujikake, Takashi Yonekawa, Misako Yamagishi, Makoto Inagami, Fumiya Kinoshita, Hirofumi Aoki, and Hitoshi Kanamori. 2017 Driver agent for encouraging safe driving behavior for the elderly. In Proceedings of the HAI 5th International Conference on Human-Agent Interaction (HAI '17). ACM, New York, 71-79. https://doi.org/10.1145/3125739.3125743

[4] Penelope Brown and Stephen C. Levinson. 1987. Politeness: Some universals in language usage. Cambridge University Press, Cambridge.

[5] Tomoki Miyamoto, Daisuke Katagami, Yuka Shigemitsu, Mayumi Usami, Takahiro Tanaka, Hitoshi Kanamori, Yuki Yoshihara, and Kazuhiro Fujikake. 2021. Influence of social distance expressed by driving support agent's utterance on psychological acceptability. Frontiers in Psychology. 12, Article 526942 (February 2021),14 pages. https://doi.org/10.3389/fpsyg.2021.526942

[6] Tomoki Miyamoto, Daisuke Katagami, Yuka Shigemitsu, Mayumi Usami, Takahiro Tanaka, Hitoshi Kanamori, Yuki Yoshihara, and Kazuhiro Fujikake. 2019. Proposal of driving support agent which speak based on politeness theory. In Proceedings of the 2019 MobiTAS Conference on HCI in Mobility, Transport and Automotive Systems, Held as Part of the 21st HCI Conference on HumanComputer Interaction, Springer, Cham, 235-244. https://doi.org/10.1007/978-3030-22666-4_17

[7] Jae-Gil Lee, Kwan Min Lee, and Seoung-Ho Ryu. 2019. Vehicle politeness in driving situations. Future Internet. 11, 2, Article 48 (February 2019), 10 pages. https://doi.org/10.3390/fi11020048
[8] Masato Takiura. 2008. Honorifics seen from politeness, politeness seen from honorifics: an overview focusing on their pragmatic relativity. Japanese Journal of Language in Society. 11, 1 (August 2008), 23-38. https://doi.org/10.19024/jajls. 11.123

[9] Srinivasan Vasant and Leila Takayama. 2016. Help me please: Robot politeness strategies for soliciting help from humans. In Proceedings of the $2016 \mathrm{CHI}$ conference on human factors in computing systems (CHI '16). ACM, New York, 4945-4955. https://doi.org/10.1145/2858036.2858217

[10] Cristen Torrey, Suan Fussell, and Sara Kiesler. 2013. How a robot should give advice. In Proceedings of the 2013 HRI Conference on Human- Robot Interaction (HRI '13), IEEE, Christchurch, 275-282. https://doi.org/10.1109/HRI.2013.6483599

[11] Tomoki Miyamoto, Daisuke Katagami, and Yuka Shigemitsu. 2017. Improving relationships based on positive politeness between humans and life-like agents. In Proceedings of the HAI 5th International Conference on Human-Agent Interaction (HAI '17). ACM, New York, 451-455. https://doi.org/10.1145/3125739.3132585

[12] Sahba Zojaji, Christopher Peters, and Catherine Pelachaud. 2020. Influence of virtual agent politeness behaviors on how users join small conversational groups. In Proceedings of the 20th ACM International Conference on Intelligent Virtual Agents (IVA '20). ACM, New York, 1-8. https://doi.org/10.1145/3383652.3423917

[13] Shuling Li, Tingru Zhang, Na Liu, Wei Zhang, Da Tao, and Ziqi Wang. 2020. Drivers' attitudes, preference, and acceptance of in-vehicle anger intervention systems and their relationships to demographic and personality characteristics. International Journal of Industrial Ergonomics, 75, Article 102899 (January 2020), 12 pages. https://doi.org/10.1016/j.ergon.2019.102899

[14] Myounghoon Jeon, Bruce N. Walker, and Thomas M. Gable. 2015. The effects of social interactions with in-vehicle agents on a driver's anger level, driving performance, situation awareness, and perceived workload. Applied Ergonomics, 50 (September 2015), 185-199. https://doi.org/10.1016/j.apergo.2015.03.015

[15] Michael Braun and Florian Alt. 2020. Identifying personality dimensions for characters of digital agents. In: El Bolock A., Abdelrahman Y. and Abdennadher S. (eds.) Character Computing. Human-Computer Interaction Series. Springer, Cham, 123-137. https://doi.org/10.1007/978-3-030-15954-2_8

[16] Boris Galitsky. 2021. Adjusting chatbot conversation to user personality and mood. Artificial Intelligence for Customer Relationship Management. HumanComputer Interaction Series. Springer, Cham, 93-127. https://doi.org/10.1007/9783-030-61641-0 3

[17] Motoki Iwashita and Daisuke Katagami. 2020. Psychological effects of compliment expressions by communication robots on humans. In Proceedings of the 2020 International Joint Conference on Neural Networks (IJCNN '20). IEEE, Christchurch, 1-8. https://doi.org/10.1109/IJCNN48605.2020.9206898

[18] Alexandra D. Kaplan, Tracy Sanders, and Peter A. Hancock. 2019. The relationship between extroversion and the tendency to anthropomorphize robots: A Bayesian analysis. Frontiers in Robotics and AI. 5, Article 135 (January 2019), 9 pages. https://doi.org/10.3389/frobt.2018.00135

[19] Jianlong Zhou, Luo Simon, and Fang Chen. 2020. Effects of personality traits on user trust in human-machine collaborations. Journal on Multimodal User Interfaces. 14 (May 2020) 387-400. https://doi.org/10.1007/s12193-020-00329-9

[20] Connor Esterwood and Lionel P. Robert. 2020. Personality in healthcare human robot interaction (H-HRI): A literature review and brief critique. In Proceedings of the 8th International Conference on Human-Agent Interaction (HAI '20). ACM, New York, 87-95. https://doi.org/10.1145/3406499.3415075

[21] Samuel D. Gosling, Peter J. Rentfrow, and William B. Swann Jr. 2003. A very brief measure of the Big-Five personality domains. Journal of Research in Personality, 37 (December 2003), 504-528. https://doi.org/10.1016/S0092-6566(03)00046-1

[22] Atsushi Oshio, Shingo Abe, and Pino Cutrone. 2012.Development, reliability, and validity of the Japanese version of ten item personality inventory (TIPI-J). The Japanese Journal of Personality. 21,1 (July 2012), 40-52. https://doi.org/10.2132/ personality.21.40 (In Japanese)

[23] Joy Paul Guilford. 1959. Three faces of intellect, American Psychologist, 14, 8, 469-479. https://doi.org/10.1037/h0046827

[24] Ewa Luger and Abigail Sellen. 2016. "Like having a really bad PA": the gulf between user expectation and experience of conversational agents. In Proceedings of the 2016 CHI Conference on Human Factors in Computing Systems (CHI '2016). ACM, New York, 5286-5297. https://doi.org/10.1145/2858036.2858288

[25] David Premack and Guy Woodruff. 1978. Does the chimpanzee have a theory of mind? Behavioral and Brain Sciences. 1 (December 1978), 515-526. https: //doi.org/10.1017/S0140525X00076512

[26] Daniel Clement Dennett. 1989. The Intentional Stance. MIT Press, Cambridge.

[27] Byron Reeves and Clifford Nass. 1996. The media equation: how people treat computers, television, and new media like real people and places. Cambridge University Press, New York.

[28] Takafumi Sakamoto and Yugo Takeuchi. 2020. Classification and prospects for HAI research based on a tentative framework. In Proceedings of the 8th International Conference on Human-Agent Interaction (HAI '20). ACM, New York, 157-162. https://doi.org/10.1145/3406499.3415085

[29] Tatsuya Nomura. 2017. Cultural differences in social acceptance of robots. In Proceedings of the 26th IEEE International Symposium on Robot and Human 
Interactive Communication (RO-MAN '17). IEEE, Christchurch, 534-538. https: //doi.org/10.1109/ROMAN.2017.8172354.

[30] Tomoko Koda and Yuuki Takeda. 2018. Perception of culture-specific gaze behaviors of agents and gender effects. In Proceedings of the 6th International Conference on Human-Agent Interaction (HAI '18). ACM, New York, 138-143. https://doi.org/10.1145/3284432.3284472

[31] Christoph Bartneck, Tomohiro Suzuki, Takayuki Kanda, and Tatsuya Nomura 2007. The influence of people's culture and prior experiences with Aibo on their attitude towards robots. AI \& Society, 21 (May 2006), 217-230. https://doi.org/10. 1007/s00146-006-0052-7

[32] Nihan Karatas, Shintaro Tamura, Momoko Fushiki, and Michio Okada. 2019. Improving human-autonomous car interaction through gaze following behaviors of driving agents. Transactions of the Japanese Society for Artificial Intelligence 34, 2 (March 2019). A-IA1_1-111. https://doi.org/10.1527/tjsai.A-IA1

[33] Jin Xu and Ayanna Howard. 2020. How much do you trust your self-driving car? Exploring human-robot trust in high-risk scenarios. 2020 IEEE International Conference on Systems, Man, and Cybernetics (SMC '20). IEEE, Christchurch, 4273-4280. https://doi.org/10.1109/SMC42975.2020.9282866.
[34] Takahiro Tanaka, Kazuhiro Fujikake, Takashi Yonekawa, Misako Yamagishi, Makoto Inagami, Fumiya Kinoshita, Hirofumi Aoki, and Hitoshi Kanamori. 2018. Study on driver agent based on analysis of driving instruction data -Driver agent for encouraging safe driving behavior (1)-. IEICE Transactions on Information and Systems. E101-D, 5 (May 2018), 1401-1409. https://doi.org/10.1587/transinf. 2017EDP7203

[35] Vanessa Tobisch, Markus Funk, and Adam Emfield. 2020. Dealing with input uncertainty in automotive voice assistants. In Proceedings of the 12th International Conference on Automotive User Interfaces and Interactive Vehicular Applications (AutomotiveUI '20). Association for Computing Machinery, ACM, New York, 161-168. https://doi.org/10.1145/3409120.3410660

[36] Cristian Danescu-Niculescu-Mizil, Moritz Sudhof, Dan Jurafsky, Jure Leskovec, and Christopher Potts. 2013. A computational approach to politeness with application to social factors. In Proceedings of the 51st Annual Meeting of the Association for Computational Linguistics (ACL '2013), ACL, 250-259. https: //www.aclweb.org/anthology/P13-1025

[37] Tong Niu and Mohit Bansal. Polite dialogue generation without parallel data. 2018. Transactions of the Association for Computational Linguistics. 6 (May 2018), 373-389. https://doi.org/373-389. 10.1162/tacl_a_00027 